\title{
Extracellular vesicles trigger ATP release and promote migration of human microglia through the P2X4 receptor / MFG-E8 - dependent mechanisms
}

Ugnè Jonavičè $\dot{e}^{1}$, Diana Romenskaja ${ }^{1}$, Karolina Kriaučiūnaité ${ }^{1}$, Akvilè Jarmalavičiūte ${ }^{1}$, Justina Pajarskiené $^{1}$, Vytautas Kašèta ${ }^{1}$, Virginijus Tunaitis ${ }^{1}$, Tarja Malm² ${ }^{2}$ Rashid Giniatulin ${ }^{2}$ and Augustas Pivoriūnas ${ }^{1 *}$

${ }^{1}$ Department of Stem Cell Biology, State Research Institute Centre for Innovative Medicine, LT01102, Vilnius, Lithuania

${ }^{2}$ A.I. Virtanen Institute for Molecular Sciences, University of Eastern Finland, Kuopio, Finland.

Send all correspondence to:

A. Pivoriūnas; email: augustas.pivoriunas@imcentras.lt

\begin{abstract}
Extracellular vesicles (EVs) effectively suppress neuroinflammation and induce neuroprotective effects in different disease models. However, the mechanisms by which EVs regulate neuroinflammatory response of microglia remain largely unexplored. Here, we addressed this issue by testing the action of EVs derived from human exfoliated deciduous teeth stem cells (SHEDs) on immortalized human microglial cells.
\end{abstract}

We found that EVs induced a rapid increase in intracellular $\mathrm{Ca}^{2+}$ and promoted a significant ATP release in microglial after 20 min of treatment. Boyden chamber assays revealed that EVs promoted microglial migration by $20 \%$. Pharmacological inhibition of different subtypes of purinergic receptors demonstrated that EVs activated microglial migration preferentially through the P2X4R pathway. Proximity ligation and co-immunoprecipitation assays revealed that EVs promote association between milk fat globule-epidermal growth factor-factor VIII (MFG-E8) and P2X4 receptor proteins. Furthermore, pharmacological inhibition of $\alpha \mathrm{V} \beta 3 / \alpha \mathrm{V} \beta 5$ integrin suppressed EV induced cell migration and formation of lipid rafts in microglia. These results demonstrate that EVs promote microglial motility through P2X4 R/ MFG-E8 - dependent mechanisms.

Our findings provide novel insights into the molecular mechanisms through which EVs target human microglia that may be exploited for the development of new therapeutic strategies targeting disease associated neuroinflammation.

Keywords: Microglia; Extracellular vesicles; migration; ATP; P2X4 receptor; MFG-E8; lipid rafts. 


\section{Introduction}

Microglia regulate immune homeostasis in the central nervous system (CNS) by initiating, maintaining and terminating neuroinflammatory response to all types of injuries [1]. Dysregulated microglial response is important for the development and propagation of various neurological disorders and therefore targeting of neuroinflammatory microglia has been considered as a promising therapeutic approach [2,3]. Extracellular vesicles (EVs) containing multiple proteins, RNAs, lipids and metabolites [4] are capable of crossing blood-brain barrier (BBB) and thus can be delivered into the brain by minimally invasive intranasal route [5-7]. Intranasal delivery of EVs has been shown to suppress neuroinflammation and promote neuroprotection in a different experimental models [8-12]. EVs specifically target and accumulate in pathologically affected areas of the brain $[6,13]$. Intranasally administered curcumin-encapsulated exosomes are selectively taken up by microglia and suppress secretion of pro-inflammatory cytokines in LPS-treated mice [9]. EVs also reduce microglial activation and prevent the induction of plethora of pro-inflammatory cytokines after status epilepticus in mice [8]. However, the mechanisms by which EVs regulate microglial responses remain largely unexplored.

During CNS injury damaged cells release several signaling molecules, including extracellular adenosine triphosphate (eATP) nucleotides that induce recruitment of microglia to the site of the injury and subsequent phagocytosis of apoptotic cells and neuronal debris. ATP potentiates bacterial killing by macrophages through purinergic P2X4 receptors by increasing the production of mitochondrial reactive oxygen species (ROS) [14]. Blockade of P2X4R signaling inhibits myelin phagocytosis in the experimental autoimmune encephalomyelitis (EAE) model [15]. Purinergic signaling is also crucial for ATP-induced chemotaxis $[16,17]$. Disruption of local ATP microgradients associated with neuronal hyperactivity during epilepsy impairs microglial motility and phagocytosis [18]. Microglia not only sense eATP by multiple purinergic receptors but can also release ATP by exocytosis [19]. Stimulation of Toll - like receptors (TLRs) triggers $\mathrm{Ca}^{2+}$ dependent release of ATP from microglia [19] and macrophages [20]. It has therefore been suggested that controlled ATP release by inflammatory cells may be used to fine-tune autocrine /paracrine responses during acute and chronic inflammation [21]. We have previously reported that EVs derived from stem cells from the dental pulp of human exfoliated deciduous teeth (SHEDs) upregulates human microglia phagocytosis [22]. In the present study we show that EVs increase intracellular $\mathrm{Ca} 2+$, trigger ATP release and significantly enhance microglial motility through milk fat globule-epidermal growth factor-factor VIII (MFG-E8)/ P2X4 receptor - 
dependent mechanisms. Our findings provide novel insights into the molecular mechanisms through which EVs target human microglia.

\section{Results}

\section{Characterization of EVs}

Transmission electron microscopy (TEM) of EV samples isolated from SHEDs identified vesicles with a typical cup - shaped morphology (Fig. 1A), nanoparticle tracking analysis showed that size distribution of the EVs was around $150 \mathrm{~nm}$ (Fig.1B). Western blot analyses revealed that EV fractions were positive for the Milk fat globule-epidermal growth factor-factor VIII (MFG-E8) (Fig1C).

A

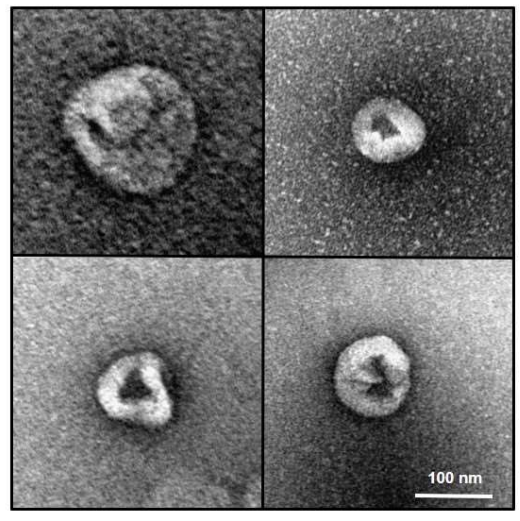

B

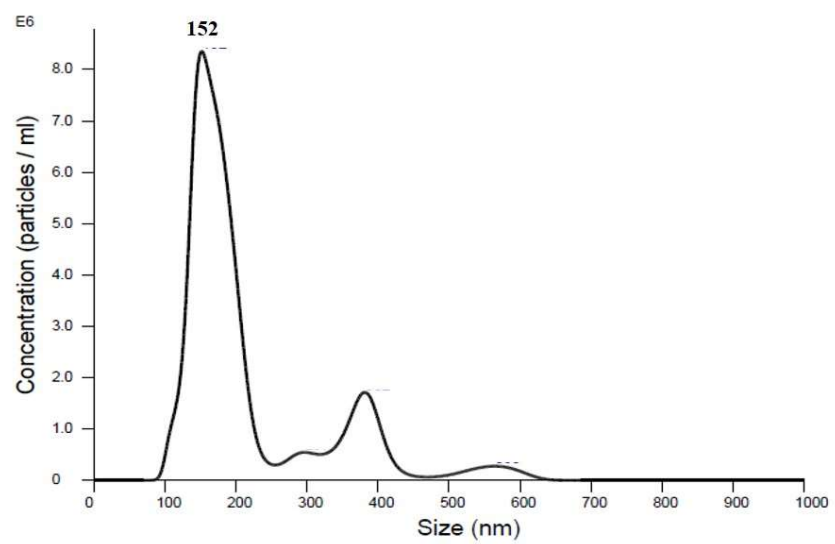

$\mathrm{C}$

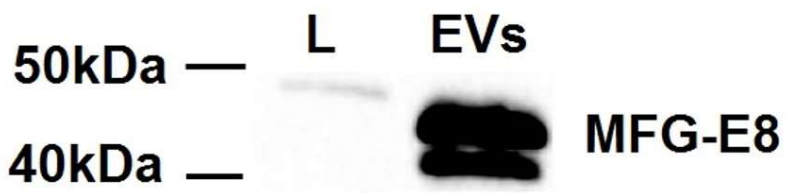

Figure 1. Characterization of extracellular vesicles (EVs) isolated from stem cells from the dental pulp of human exfoliated deciduous teeth (SHEDs). (A) Transmission electron microscopy of EVs isolated from SHEDs $(\times 120,000$ magnification). (B) Concentration and particle size of EVs derived from SHEDs as analysed by nanoparticle tracking analysis using NanoSight LM10 instrument (Malvern Panalytical). Size distribution of the EVs was around $150 \mathrm{~nm}$. (C) Samples from the cell lysates (L) and extracellular vesicles (EVs) were subjected to electrophoresis, blotted and the membrane was probed with antibodies against MFG-E8. Bands were visualized by incubation with appropriate horseradish peroxidase-conjugated secondary antibodies and chemiluminescence substrate. Full blot is available in the Supplementary Figure 1. 


\section{EVs increase intracellular $\mathrm{Ca}^{2+}$ levels in human microglia}

Increase in mobilization of intracellular $\mathrm{Ca}^{2+}$ triggers many functions of microglia including activation, motility and release of ATP $[19,20]$. We used live calcium imaging to test how shortterm treatment of human microglia with EVs isolated from SHEDs alter microglial intracellular $\mathrm{Ca}^{2+}$ levels (Fig 2). Our data show that acute $1 \mathrm{~min}$ application with EVs (4 AU/ml) significantly increased $\mathrm{Ca}^{2+}$ levels in microglia (by 3.6 folds when compared to baseline level (EV-free $\mathrm{BS}$ ); $\mathrm{p}<$ 0.0001, (Fig. 2C).

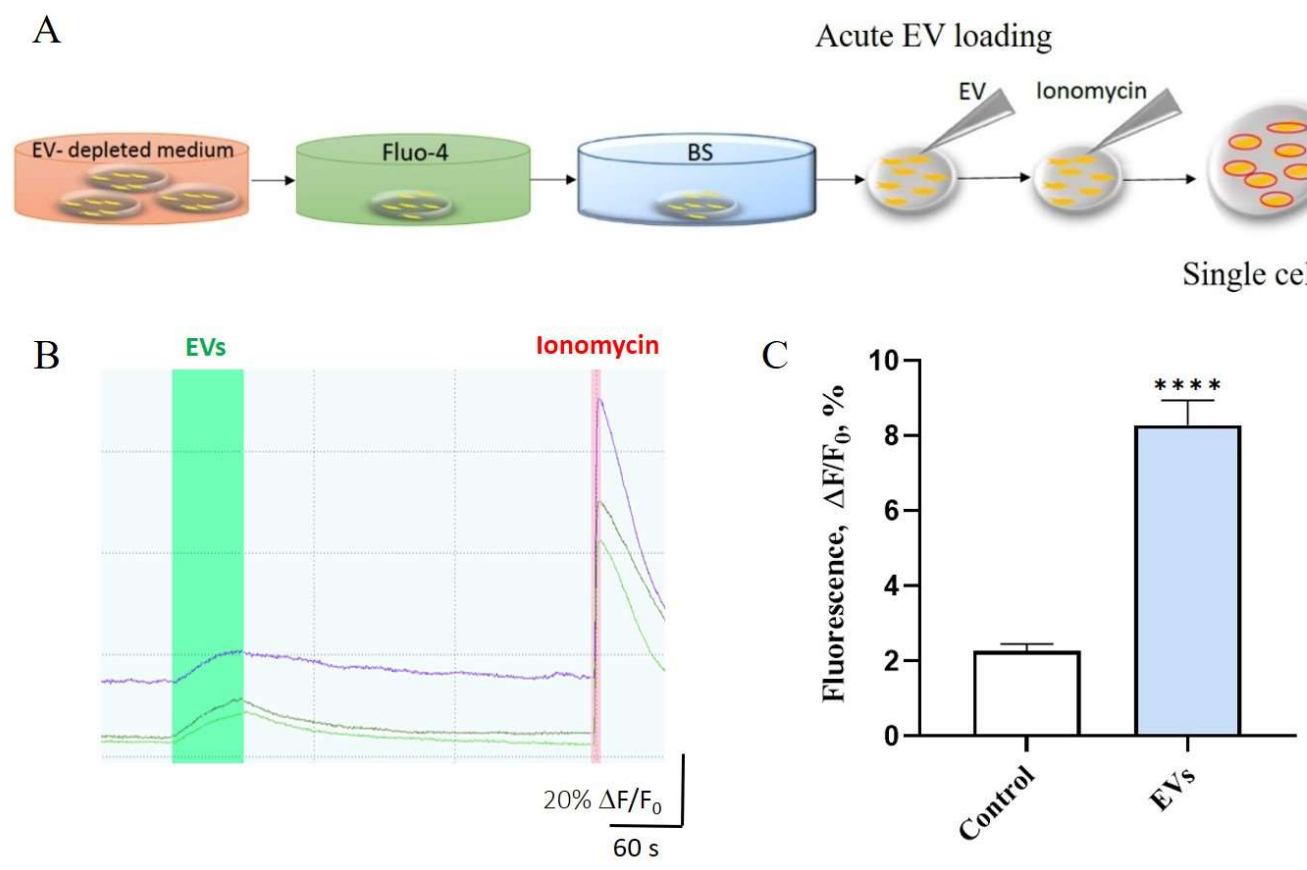

Figure 2. EVs increase intracellular $\mathrm{Ca}^{2+}$ in human microglial cells. (A) Experimental design. $(\mathrm{B}, \mathrm{C})$ Response of human microglial cells to acute 1 min treatment of EVs. Intracellular $\mathrm{Ca}^{2+}$ concentrations in single cells were visualized with the sensitive imaging system (Till Photonics, Germany). The peak of fluorescence in each individual cell during EV treatment was normalized to peak of fluorescence after response to ionomycin and presented as a percent of intracellular calcium signal. The graph represent mean \pm SEM, statistically significant difference was determined by Mann-Whitney test, $* * * * \mathrm{p}<0.0001$ (Control $\mathrm{N}=85$ cells, EVs; $\mathrm{N}=127$ cells; cells from 3 experiments). 


\section{EVs trigger ATP release in human microglia}

Different biologically relevant stimuli can induce microglial ATP release [19]. We therefore investigated levels of the extracellular ATP (eATP) in human microglia cultures stimulated with EVs (1 AU) for 20 and 60 min. Treatment with EVs for 20 min significantly (by 2 folds; $p=$ 0.0158) increased the levels of eATP in the microglia cultures (Fig. 3). This increase was no longer evident after $60 \mathrm{~min}$ of incubation $(\mathrm{p}=0.6417)$ (Fig. 3) suggesting that that EVs induce a rapid and transient ATP release in human microglia.

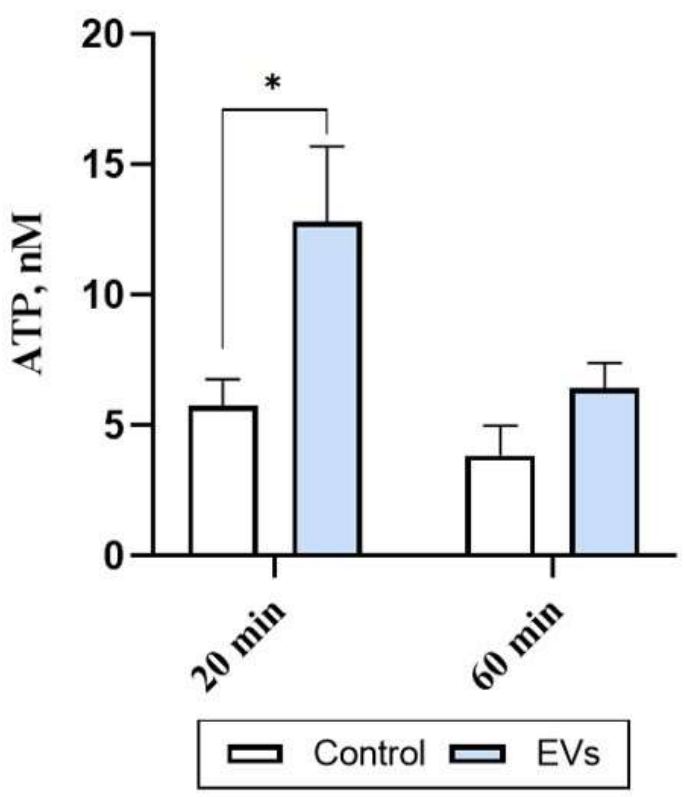

Figure 3. EVs trigger ATP release from human microglial cells. Microglial cells were exposed to EVs (1 AU/well) diluted in BS for 20 and 60 minutes. Supernatants from each well were subjected to ATP assay. ATP was measured using ATPlite Luminescence Assay System with Perkin Elmer Wallac 1420 Victor2 instrument using Wallac 1420 software. Each bar represents mean \pm SEM, statistically significant difference was determined by two-way ANOVA, Sidak's multiple comparisons test, $* \mathrm{p}<0.05$ ( $\mathrm{n}=15-20$ wells from 3 experiments).

\section{EVs promote P2X4R-dependent migration of human microglia}

It is well known that eATP serves as a powerful trigger for motility of microglia $[23,24]$. We therefore hypothesized that EV- triggered ATP release may promote microglial motility via autocrine and (or) paracrine mechanisms. Indeed, EVs significantly increased microglial migration 
by $20 \%(n=22, p=0.0001)$ in the Boyden chamber assay. (Fig.4B). Since microglia are highly sensitive to eATP through several subtypes of purinergic receptors such as P2X4 and P2Y12 [25]. we tested different inhibitors to determine which purinergic signaling pathway is responsible for the observed effects. Whilst the nonselective antagonist of ATP-gated P2 receptors suramin failed to significantly alter cellular migration ( $n=3, p=0.1321$; Fig.4C), a highly potent P2Y12 antagonist AR-C 69931 significantly decreased the migration by $30 \%(n=3, p=0.0009$; Fig. 4D). ARC69931 failed to prevent the EV induced migration ( $n=3, p=0.0002$; Fig. 4D). These results indicate that suramin and AR-C69931 did not suppress EV-induced migration of microglial cells. $\mathrm{P} 2 \mathrm{X} 4 \mathrm{R}$ is relatively insensitive to the blockade by the suramin, we therefore used a potent selective P2X4R antagonist 5-BDBD [26]. We found 5-BDBD significantly decreased microglial migration in the presence and absence of EVs $(n=5, p=0.0263)$ (Fig.4E). Interestingly, EVs also neutralized inhibitory effect of ATP degrading enzyme apyrase on the migration of microglia $(n=3, p=$ 0.0001) (Fig. 4F). Taken together our data indicate that EVs activate microglial migration via P2X4R pathway and that this effect does not depend on the EV-triggered ATP release. 
A

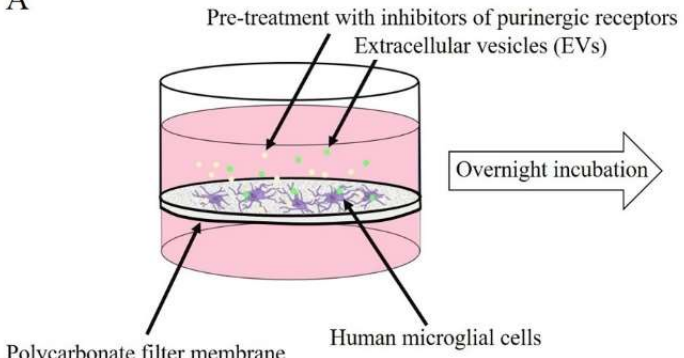

Polycarbonate filter membrane

$\mathrm{C}$

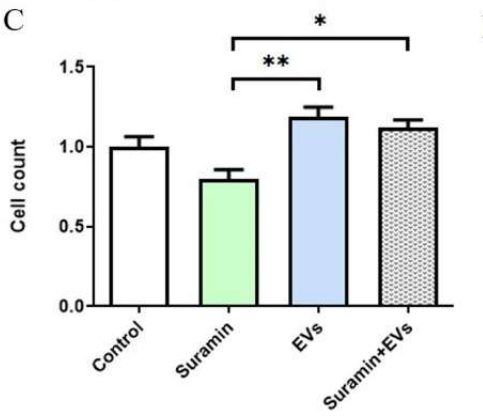

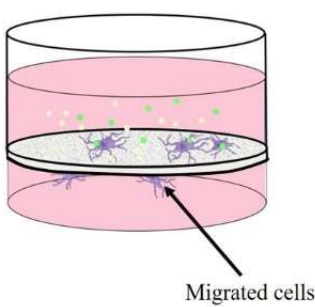

$\mathrm{B}$

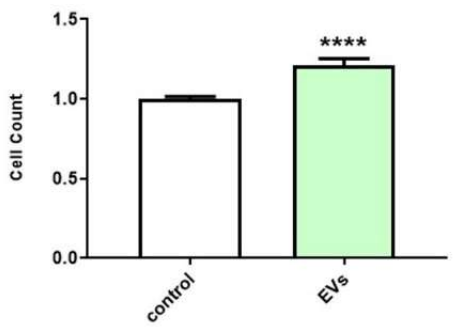

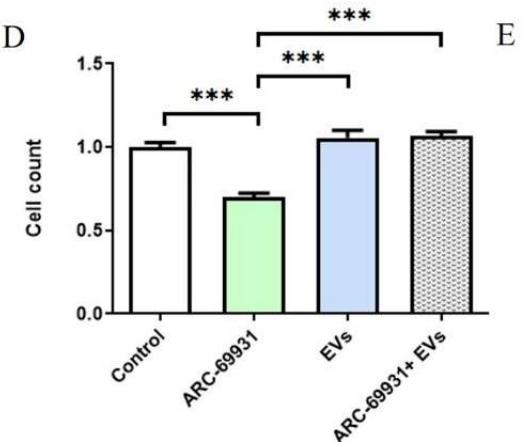

$\mathrm{E}$

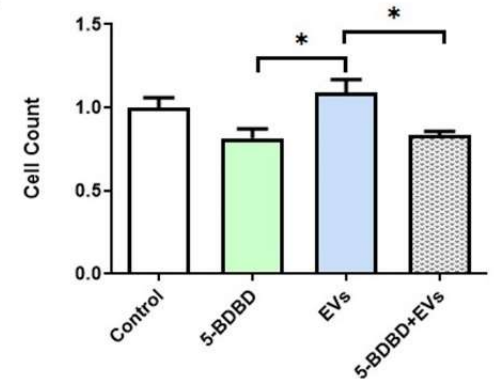

F

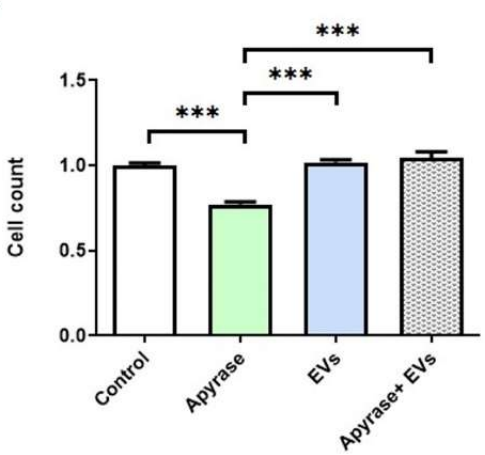

Figure 4. EVs promote migration of microglia through the $\mathrm{P} 2 \mathrm{X} 4$ receptor - dependent mechanisms. (A) Migration assays were performed in the Boyden 10 well chemotaxis chamber (Neuro Probe) using $8 \mu \mathrm{m}$ pore polycarbonate membranes. Microglial cells were plated into the upper wells, pre-treated for 30 min with inhibitors against different subtypes of purinergic receptors, treated with $1 \mathrm{AU}$ of EVs and then left overnight. The number of cells that transmigrated to the opposite side of the membrane was determined using optical microscope in three randomly chosen fields from at least three wells for each experimental group. (B) EVs promote migration of human microglial cells. Data represent mean \pm SEM values, Welch's test, $* * * * p<0.0001 ; \mathrm{n}=22$; (C) The effects of nonselective antagonist of ATP-gated P2 receptors suramin $(200 \mu \mathrm{M})$; (D) inhibitor of P2Y12 AR-C69931 (1 $\mu \mathrm{M})$; (E) selective inhibitor of P2X4R 5-BDBD (10 $\mu \mathrm{M})$; (F) ATP degrading enzyme apyrase $(20 \mathrm{U} / \mathrm{ml})$. For these experiments apyrase and $1 \mathrm{AU}$ of EVs were added simultaneously to the upper wells and cells incubated overnight. Data represent measurements from three independent experiments (only treatment with 5-BDBD from five). The graph represent mean $\pm \mathrm{SD}$ values, groups were compared with one-way ANOVA, Tukey's multiple comparisons test, ${ }^{*} \mathrm{p}<0.05,{ }^{*} \mathrm{p}<0,01,{ }^{* * *} \mathrm{p}<0.001$. 


\section{EVs promote association between MFG-E8 and P2X4 receptor proteins in human microglia}

Milk fat globule-epidermal growth factor-factor VIII (MFG-E8) is a secretory glycoprotein expressed in microglia [27]. MFG-E8 also associates with EVs by binding to the phosphatidylserine (PS) [28, 29]. Indeed, EVs derived from the SHEDs express high levels of MFG-E8 [22] (Fig.1C). We used proximity ligation assay (PLA) to detect protein - protein interactions between MFG-E8 and P2X4 receptor in the human microglial cells. Our results show an association between MFG-E8 and P2X4 receptor proteins in control (EV-untreated) cells. Exposure to EVs for 2 hours significantly (by 4.3 folds compared to control; $\mathrm{p}<0.0001$ ) promoted association between MFG-E8 and P2X4 receptor proteins (Fig.5) suggesting a close association between MFG-E8 and P2X4 receptor proteins in human microglia which is significantly promoted in cells exposed to EVs.
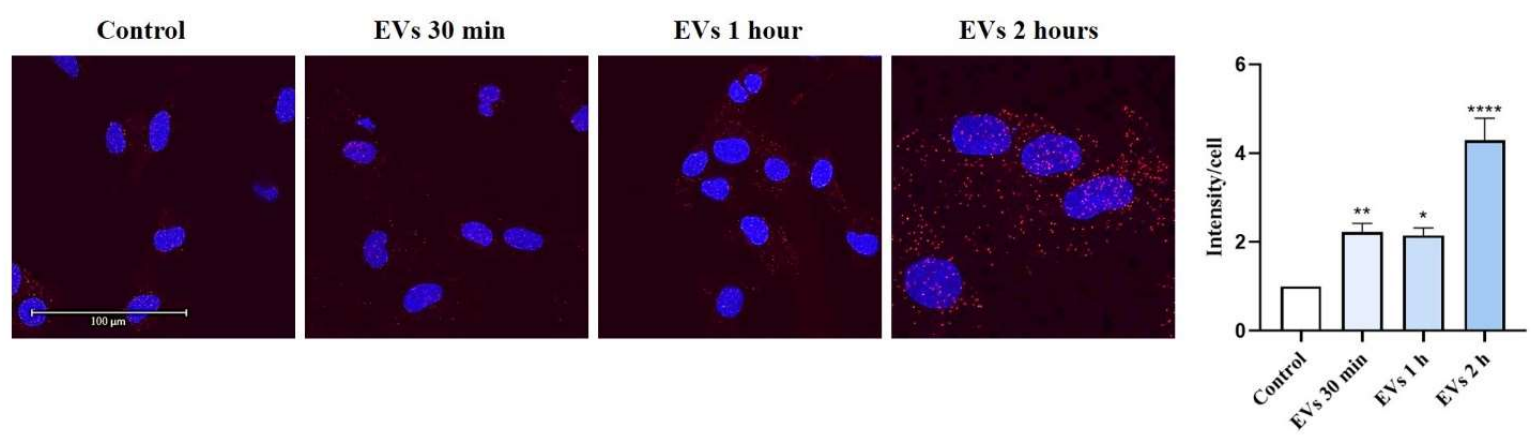

Figure 5. EVs promote association between MFG-E8 and P2X4 receptor proteins in human microglia. (A) Confocal images of proximity ligation assay (PLA) dots (red) in human microglial cells. The cells were treated with 1AU of EVs for $30 \mathrm{~min}, 1 \mathrm{~h}$ and $2 \mathrm{~h}$ before PLA was conducted. Nuclei, DAPI (blue). Scale bar $=100 \mu \mathrm{m}$. (B) PLA dots per cell were counted with ImageJ program. Data shown represent results of 10 fields of view for each experimental group from three independent biological experiments Data represents mean $\pm \mathrm{SEM}, * \mathrm{p}<0.05$; $\mathrm{N}=3$; KruskalWallis, Dunn's multiple comparisons test.

Co-immunoprecipitation assays also confirmed association between MFG-E8 and P2X4 receptor proteins in the microglial cells (Fig.6).

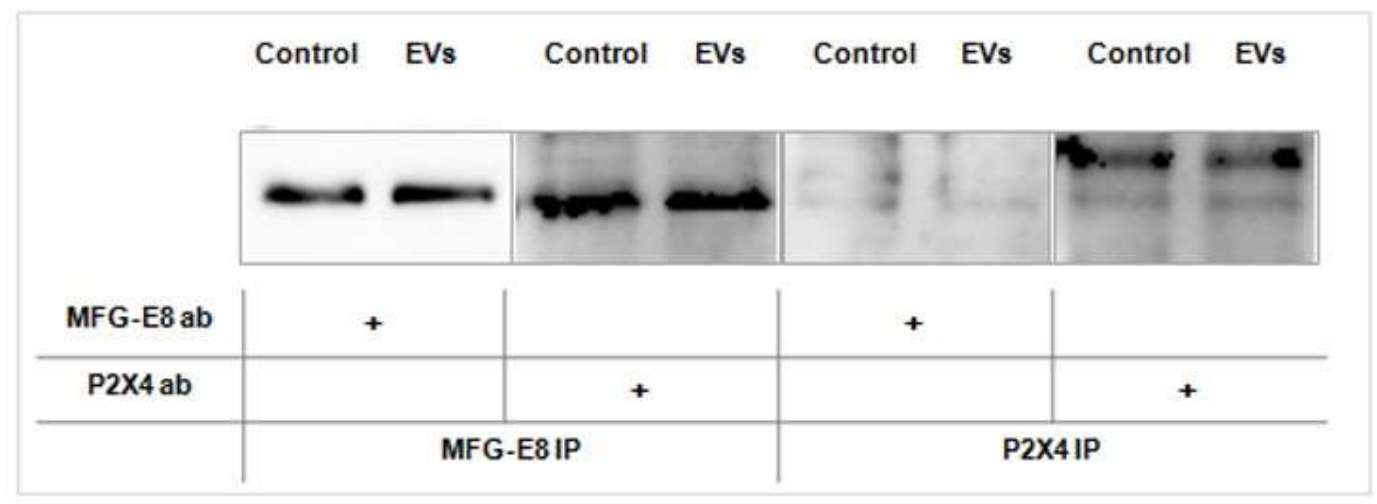


Figure 6. Co-immunoprecipitation of MFG-E8 and P2X4. Representative western blots showing co-immunoprecipitation of MFG-E8 and P2X4 receptor proteins in human microglial cells. Full blots are available in Supplementary Figure 2.

Inhibition of MFG-E8 receptor with cilengitide suppressed EV - induced migration and formation of lipid rafts in microglia

MFG-E8 protein interacts with target cells through $\alpha_{\mathrm{v}} \beta_{3}$ and $\alpha_{\mathrm{v}} \beta_{5}$ integrins [27]. Cilengitide selectively blocks activation of the $\alpha_{\mathrm{v}} \beta_{3}$ and $\alpha_{\mathrm{v}} \beta_{5}$ integrins and is effectively used as an inhibitor of MFG-E8 signaling [30, 31]. We therefore tested the effects of cilengitide on EV - induced migration of microglia. Pretreament with $10 \mu \mathrm{M}$ of cilengitide significantly suppressed EV - induced migration of microglia (by $17 \%$ compared to control; $n=8 ; p=0.0001$ ) (Fig.7).

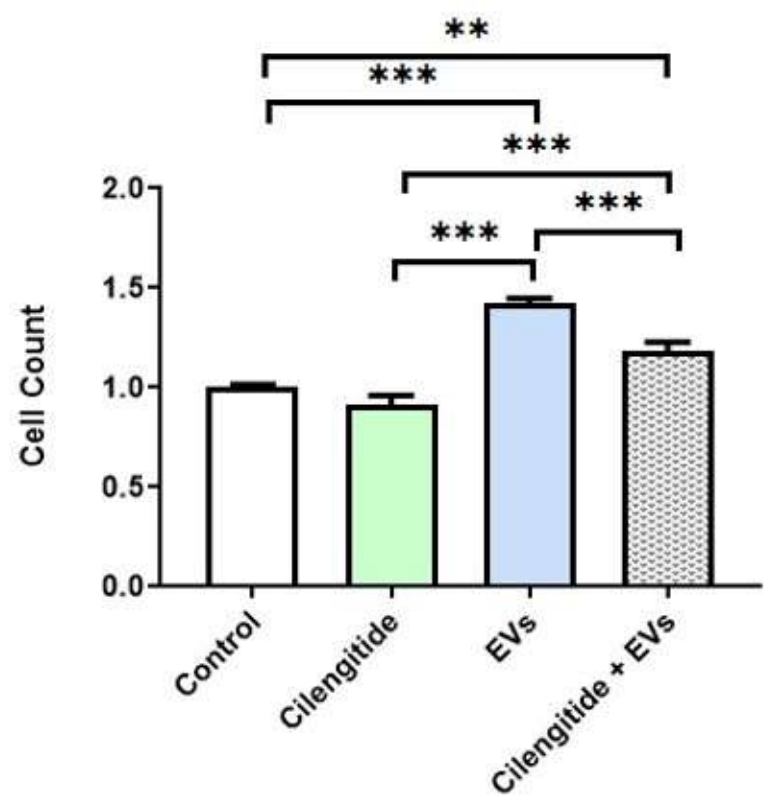

Figure 7. Inhibition of MFG-E8 receptor with cilengitide suppressed EV - induced migration of microglia. For migration experiments (see above) microglial cells were pre-treated for 30 min with inhibitor MFG-E8 receptor cilengitide $(10 \mu \mathrm{M})$, then treated with 1 AU of EVs. Data represents mean $\pm \mathrm{SD}, * * \mathrm{p}<0.01,{ }^{* * *} \mathrm{p}<0.001 ; \mathrm{N}=3$; One way ANOVA, Tukey's multiple comparisons test.

Lipid rafts serve as organizing platforms enriched in different signaling proteins and receptors initiating inflammatory signaling and different cellular responses [32]. We tested how EVs affect formation of lipid rafts in the microglia. Treatment of human microglia with EVs for 30 min 
significantly increased lipid raft formation (by $113 \%$ compared to control; $n=3 ; p=0.0001$ ) (Fig.8). Again, pretreatment with cilengitide significantly (by $44 \%$ compared to control; $n=3 ; p=0.0002$ ) suppressed EV - induced lipid raft formation (Fig.8).
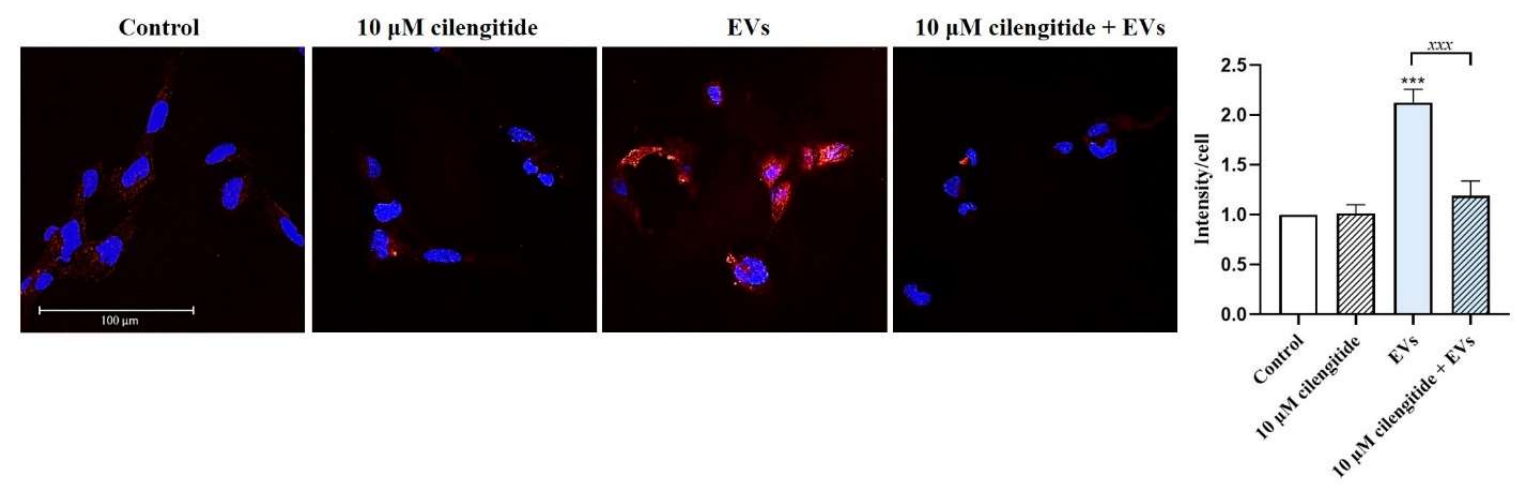

Figure 8. Inhibition of MFG-E8 receptor with cilengitide suppressed EV - induced formation of lipid rafts. (A) Confocal images of lipid rafts (red) expressing human microglial cells. The cells were pre-treated with $10 \mu \mathrm{M}$ cilengitide for $2 \mathrm{~h}$ and then treated with EVs (1 AU) of EVs for $30 \mathrm{~min}$ before lipid raft labeling and fixation. Lipid rafts were labeled with Vybrant ${ }^{\circledR}$ Alexa Fluor ${ }^{\circledR} 594$ Lipid Raft Labeling Kit (Thermo Fisher Scientific) according to the manufacturer's protocol. Nuclei, DAPI (blue). Scale bar $=100 \mu \mathrm{m}$. (B) The mean fluorescence intensity of lipid rafts per cell were measured with Leica Application Suite X (LAS X) software. Data shown represent results of 15 fields of view for each experimental group from three independent biological experiments $(n=3)$ plotted as mean \pm SEM. Statistical significance was analyzed by Kruskal-Wallis test using Dunn's multiple comparison post-hoc test. ${ }^{* *}, \mathrm{xxx}, \mathrm{p}<0.001$;

\section{Discussion}

In this study we demonstrate for the first time that EVs trigger ATP release and promote migration of human microglia through the P2X4 receptor/MFG-E8 - dependent mechanisms. Our findings provide novel insights into the molecular mechanisms through which EVs target human microglia. Our initial observation that EVs increased intracellular $\mathrm{Ca}^{2+}$ levels and induced a rapid ATP release in microglia prompted us to test whether these effects were responsible for the increased microglial motility. Blockage of P2X4 receptor with selective inhibitor 5-BDBD prevented EV - induced increase in microglial migration. However, our findings do not support the model according to which EV- triggered ATP release promote microglial motility via autocrine and (or) paracrine mechanisms. First of all, use of ATP degrading enzyme apyrase did not suppress the effects of EVs on the microglial migration. Furthermore, it is unlikely, that EV - induced transient (20 min) 
increase of eATP could significantly affect overnight migration of cells through the membrane in the Boyden chamber unless it triggers early remodeling supporting formation of the cell migration mechanisms. Several reports have demonstrated that MFG-E8 secretory glycoprotein is highly expressed in the different types of EVs. By its discoidin domain MFG-E8 protein associates with PS exposed on the membranes and this property has already been used for the in vivo identification of apoptotic and EV-bound cells [29]. EVs used in our study expressed high levels of MFG-E8 protein (Fig. 1C). We therefore tested whether MFG-E8 and P2X4 receptor proteins directly interact in human microglia. Indeed, sensitive in situ proximity ligation assays (PLA) revealed a close association between MFG-E8 and P2X4 receptor proteins in EV - treated and untreated cells (Fig.5). Microglial cells constitutively express and secrete MFG-E8 protein [27, 33], it has therefore been impossible to distinguish between microglial and vesicular MFG-E8 fractions. Nevertheless, treatment with EVs remarkably promoted association between MFG-E8 and P2X4 receptor proteins (Fig.5). To our knowledge, this is the first demonstration of direct interaction between MFG-E8 and P2X4 receptor proteins. The MFG-E8 protein has epidermal growth factor domain which recognizes integrins $\alpha_{\mathrm{V}} \beta_{3}$ and $\alpha_{\mathrm{V}} \beta_{5}$ on the target cells [27]. MFG-E8 has been shown to inhibit necrotic cell-induced and ATP-dependent IL-1 $\beta$ production by macrophages through mediation of integrin $\beta_{3}$ and P2X7 receptor interactions in primed cells [34]. Several studies have also demonstrated a direct physical association between $\mathrm{P} 2 \mathrm{X} 4$ and $\mathrm{P} 2 \mathrm{X} 7$ receptors in different types of cells $[35,36]$. We therefore propose that EV - associated MFG-E8 may interact with P2X4 receptors in human microglia. This statement is further supported by our finding that cilengitide, which is a cyclized RGD-containing pentapeptide that selectively blocks activation of the $\alpha v \beta_{3}$ and $\alpha v \beta_{5}$ integrins and is effectively used as inhibitor of MFG-E8 signaling [30, 31], suppressed EV induced migration of microglia (Fig.7). P2X4R signaling is important for phagocytosis during EAE [15]. On the other hand, MFG - E8 - mediated phagocytic clearence of apoptotic cells by microglia is crucial for the proper regulation of the neuroinflammatory response in the CNS [27] [37]. We have also previously demonstrated that EVs increased phagocytic activity of human microglial cells [22]. Although in this study we did not test the effects of cilengitide on the EV - induced phagocytic activity of microglia, we propose that EVs may also promote phagocytic response through the MFG-E8 - dependent pathway. We also suggest that after therapeutic administration EVs can be selectively recognized and internalized by microglial cells through MFG-E8 $\alpha \mathrm{V} \beta 3 / \alpha \mathrm{V} \beta 5$ - dependent mechanisms. This model may at least partially explain selective EV targeting to the pathologically affected areas $[6,13]$ and specific accumulation in microglial cells [8-12]. Further studies, especially using MFG-E8 - deficient animals are needed to clarify these issues. 
Different stimuli promote formation and enlargement of lipid rafts serving as organizing platforms initiating inflammatory signaling and different cellular responses [32]. Lipid rafts are enriched in the innate immune receptors TLRs 2 and 4, TREM2, IFN $\gamma R$, purinergic receptors P2X and P2Y, integrins and other signaling proteins $[32,38,39]$. We therefore reasoned that EVs may also affect lipid raft formation in target cells. Indeed, exposure to EVs for 30 min greatly increased lipid raft formation in microglia (Fig.8). Furthermore, pretreatment with cilengitide prevented EV - induced lipid raft formation showing that EVs trigger lipid raft formation through MFG-E8 $-\alpha \mathrm{V} \beta 3 / \alpha \mathrm{V} \beta 5$ depending mechanisms.

Based on these findings, we propose a novel mechanism for the EV action on microglial cells (Fig.9).

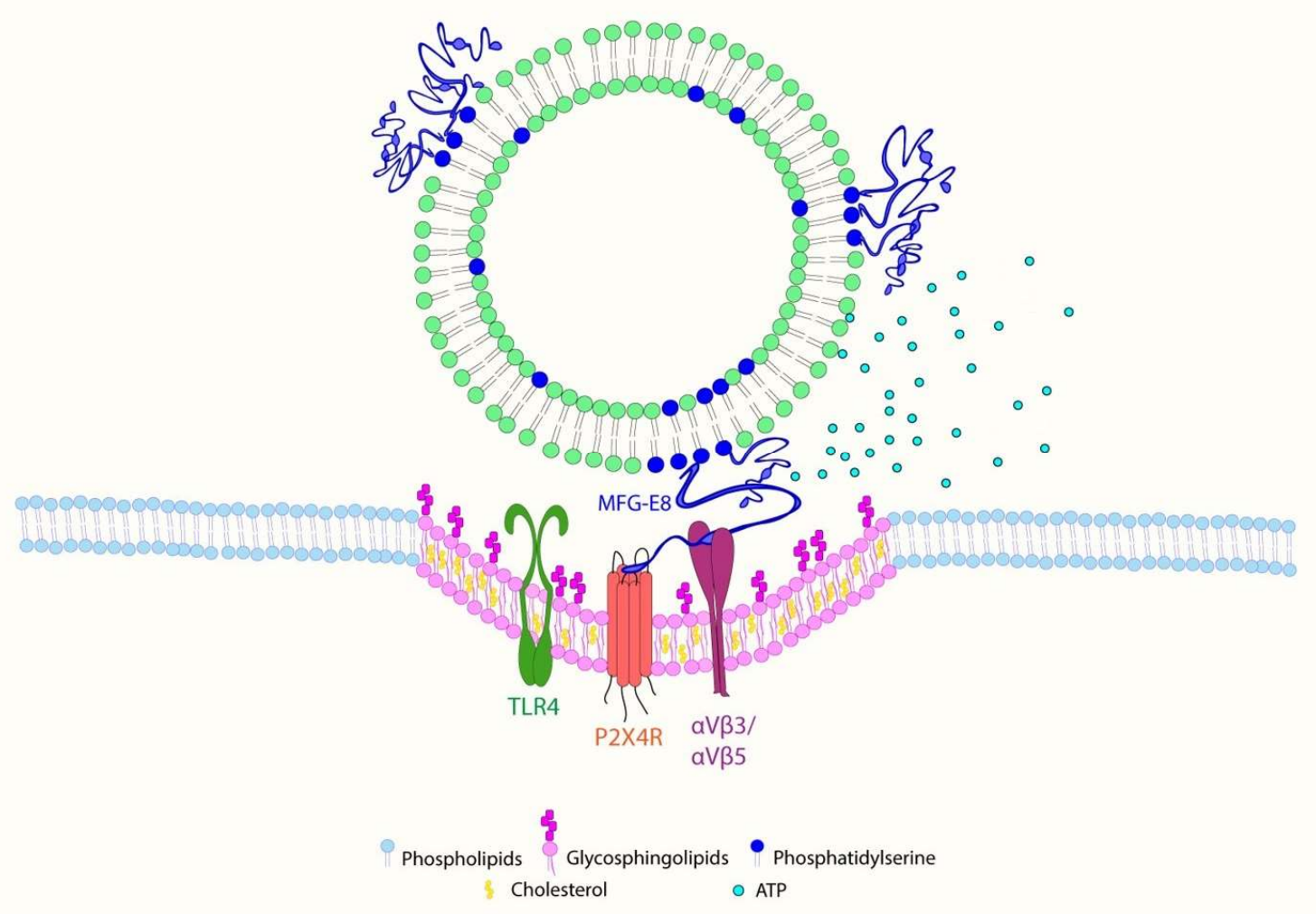

Figure 9. Proposed mechanism for the EV action on microglial cells. EVs carrying MFG-E8 proteins associated with phosphatidylserine exposed on the outer membrane are recognized by the $\alpha \mathrm{V} \beta 3 / \alpha \mathrm{V} \beta 5$ integrin receptors of microglial cells and trigger lipid raft formation, interaction with P2X4 receptors and possibly other molecules enriched in the lipid rafts such as components of TLR4 multireceptor complex. These events lead to the upregulation of intracellular $\mathrm{Ca}^{2+}$, release of ATP and increased motility of microglia.

EVs carrying MFG-E8 proteins are recognized by the $\alpha \mathrm{V} \beta 3 / \alpha \mathrm{V} \beta 5$ integrin receptors of microglial cells and trigger lipid raft formation, interaction with P2X4 receptors and possibly other molecules 
enriched in the lipid rafts such as components of TLR4 multireceptor complex. These events lead to the upregulation of intracellular $\mathrm{Ca}^{2+}$, release of ATP and increased motility of microglia. In conclusion our study demonstrates the importance of MFG-E8 - P2X4 signaling pathway for the immunomodulatory action of EVs in human microglia. Our findings could be potentially exploited for the development of new therapeutic strategies targeting neuroinflammatory microglia.

\section{Materials and Methods}

Culture of stem cells from the dental pulp of human exfoliated deciduous teeth (SHEDs) and human microglial cells

SHEDs were obtained from human exfoliated deciduous teeth of a child, whose parents had signed an informed consent. Material was collected under the approval of the Lithuanian Bioethics committee (Nr. 6B-08-173; 2008-04-22). SHEDs were isolated according previously described protocol [22]

For the isolation of extracellular vesicles (EVs) SHEDs from the 3-5rd passages were grown until the cultures reached subconfluence, then standard medium was changed to the serum-free medium MSC NutriStem XF (Biological Industries, Kibbutz Beit Haemek, Israel). Immortalized (SV40) human microglial cell line was purchased from ABM. Human microglial cells were cultivated on cell culture tissue flasks coated with $50 \mu \mathrm{g} / \mathrm{ml}$ of rat tail collagen I (Gibco) in high glucose (4.5 $\mathrm{mg} / \mathrm{ml}$ ) DMEM supplemented with glutamax (Gibco) and $10 \%$ of EV-depleted FBS (Biochrom).

\section{Isolation and characterization of extracellular vesicles}

Isolation of extracellular vesicles (EVs) was performed using differential centrifugation according to the described protocol [40] with some modifications. All centrifugation steps were performed at $4{ }^{\circ} \mathrm{C}$. Supernatants collected from SHEDs cultivated in serum-free medium MSC NutriStem XF (Biological Industries) were centrifuged successively at increasing speeds (300 g for $10 \mathrm{~min}, 2000 \mathrm{~g}$ for $10 \mathrm{~min}$, then at $20000 \mathrm{~g}$ for $30 \mathrm{~min}$ ). The final supernatants were ultracentrifuged at $100000 \mathrm{~g}$ for $70 \mathrm{~min}$ in Sorvall LYNX 6000 ultracentrifuge, with rotor T29-8x50 in oak ridge centrifuge tubes with sealing caps (all from Thermo Fisher Scientific, Rochester, NY), then the pellets were washed in $40 \mathrm{ml}$ PBS and ultracentrifuged again at $100000 \mathrm{~g}$ for $70 \mathrm{~min}$. Final pellets of EVs (exosomal fraction) were resuspended in sterile PBS and stored at $-20^{\circ} \mathrm{C}$. 
Nanoparticle tracking analysis (NTA) was performed with NanoSight LM10 instrument (Malvern Panalytical).

Transmission electron microscopy (TEM) of EVs have been performed according to the previously published protocol [40] with some modifications. First, EVs in PBS were fixed with 2\% PFA on icefor $40 \mathrm{~min}$. Then, Formvar-carbon coated copper grid was floated on a drop of fixed EV suspension for $20 \mathrm{~min}$ at room temperature. Thereafter, the grid was washed in a drop of PBS for 2 min at room temperature. After washing, the grid was incubated in a drop of $1 \%$ glutaraldehyde for $5 \mathrm{~min}$ at room temperature. Then the sample was washed 8 times for $2 \mathrm{~min}$ by transferring grid from one drop of distilled water to another. The sample was contrasted on a drop of $2 \%$ neutral uranyl acetate for $5 \mathrm{~min}$ at room temperature in the dark. Afterward, the grid was incubated on a drop of $2 \%$ methylcellulose $/ 0.4 \%$ uranyl acetate for 10 min on ice in the dark. In the end grid was taken by stainless steel loop and air-dried for $5 \mathrm{~min}$. All incubations were performed on a Parafilm sheet with the coated side of grid facing the drop. The prepared EV samples were analyzed with the TEM JEOL JEM-2100F High Resolution EM-20023 (JEOL, Freising, Germany) at $80 \mathrm{kV}$. The images were taken with a Olympus Quemesa camera, using the iTEM 5.2 software (Fig.1A). The yield of EVs prepared from the supernatants of SHEDs grown until subconfluence on the cell culture flasks (representing surface area $37.5 \mathrm{~cm}^{2}$ ) and conditioned for 72 hours in serum-free medium MSC NutriStem XF was defined as 1 activity unit (AU). According to the NTA measurements (Fig.1B) $1 \mathrm{AU}$ corresponded to the $3.65 \times 10^{8}$ of EVs for migration assays and $5.2 \mathrm{x}$ $10^{8}$ of EVs for $\mathrm{Ca}^{2+}$-imaging and ATP assays.

\section{Measurements of intracellular $\mathrm{Ca}^{2+}$ concentration}

Human microglial cells were plated on rat tail collagen type I pre-coated coverslips $\left(4000 \mathrm{cells} / \mathrm{cm}^{2}\right)$ for 24 hours in complete DMEM medium (with $10 \% \mathrm{FBS}$ and $1 \% \mathrm{P} / \mathrm{S}$ ) depleted of EVs by ultracentrifugation at $100000 \mathrm{x}$ g for 6 hours at $4{ }^{\circ} \mathrm{C}$. Medium was removed and coverslips were loaded with fresh medium containing $10 \mu \mathrm{M}$ fluo-4 AM for $30 \mathrm{~min}$ at $37^{\circ} \mathrm{C}$. After removal of Fluo4 AM solution, coverslips were moved to basic solution (BS) containing in mM: $152 \mathrm{NaCl}, 2.5$ $\mathrm{KCl}, 10 \mathrm{HEPES}, 10$ glucose, $1 \mathrm{MgCl}_{2}$ and $2 \mathrm{CaCl}_{2}$ for $10 \mathrm{~min}$ at $37^{\circ} \mathrm{C}$. Then cells were placed in the experimental chamber and continuously perfused with BS. Thereafter changes in the intracellular $\mathrm{Ca}^{2+}$ concentration in single cells were visualized with the sensitive imaging system (Till Photonics, Germany). Cells were illuminated with the excitation light $(480 \mathrm{~nm})$ and the emission light passed through the FITC filter (Chroma Tech., USA). Drugs and EVs were applied by PC-controlled fast application system Biologic, Franbce) according to the following order: BS 
for $2 \mathrm{~min}$, EVs (4 AU/ml) for $1 \mathrm{~min}$, BS for $5 \mathrm{~min}, 10 \mu \mathrm{M}$ ionomycin for $2 \mathrm{~s}$, BS for $2 \mathrm{~min}$. The peak of fluorescence in each individual cell during EV treatment was normalized to peak of fluorescence after response to ionomycin and presented as a percent of intracellular calcium signal.

\section{ATP assay}

Human microglial cells were plated in rat tail collagen type I pre-coated 24-well plate (20 000 cells/well) for 72 hours in complete DMEM medium depleted of EVs. Medium was removed, cells washed once with BS, then cells were exposed to EVs (1 AU/well) diluted in BS (200 $\mu \mathrm{l} /$ well) containing $100 \mu \mathrm{M}$ NTPDase inhibitor ARL 67156 for 20 and $60 \mathrm{~min}$. Supernatants from each well were collected, heated for 5 minutes at $65^{\circ} \mathrm{C}$ and subjected to ATP assay. ATP was measured using ATPlite Luminescence Assay System according to the manufacturer's instructions with minimal modifications: supernatants were loaded into a flat-bottom white 96 -well plate $(100 \mu \mathrm{l} / \mathrm{well})$, then substrate solution was added to supernatants $(50 \mu \mathrm{l} /$ well $)$ and plate was incubated for $10 \mathrm{~min}$ at room temperature on orbital shaker in the dark. Immediately after incubation, luminescence was measured with Perkin Elmer Wallac 1420 Victor2 instrument using Wallac 1420 software.

\section{Migration assay}

Migration assays were performed in the Boyden 10 well chemotaxis chamber (Neuro Probe), according to the manufacturer's instructions. Briefly, complete DMEM medium (supplemented with EV-depleted $10 \% \mathrm{FBS}$ and $1 \% \mathrm{P} / \mathrm{S}$ ) was added to the lower wells and separated from upper wells by a polycarbonate filter membrane with $8 \mu \mathrm{m}$ size pores. Microglial cells were plated into the upper wells (30 000 cells in $285 \mu \mathrm{l}$ of complete medium per well), left overnight and then pretreated for $30 \mathrm{~min}$ with one of the following inhibitors: $200 \mu \mathrm{M}$ suramin, $1 \mu \mathrm{M}$ AR-C66931, $10 \mu \mathrm{M}$ 5-BDBD (all from Tocris), or with $10 \mu \mathrm{M}$ of cilengitide (Sigma) for 2 hours. Afterwards cells were treated with $1 \mathrm{AU}$ of EVs and then left overnight. For the apyrase treatments, $20 \mathrm{U} / \mathrm{ml}$ apyrase (Sigma) and $1 \mathrm{AU}$ of EVs were added simultaneously to the upper wells and cells incubated overnight. After incubation membranes were washed 3 times with PBS, fixed with methanol for 5 minutes, air-dried, stained with $0.25 \%$ Cristal Violet for 15 minutes and again washed 3 times with PBS. The number of cells that have migrated to the opposite side of the membrane was determined using optical microscope in three randomly chosen fields from at least three wells for each experimental group. 


\section{Co-immunoprecipitation and Western blot analysis}

For preparation of total cell lysates, cell monolayers were washed twice with cold PBS, pH 7.3, and lysed in Pierce IP lysis buffer (Thermo Scientific) supplemented with 1x Halt protease inhibitor cocktail (Thermo Scientific) for $5 \mathrm{~min}$ on ice. Samples were centrifuged at $14000 \mathrm{~g}$ for $15 \mathrm{~min}$ at $4{ }^{\circ} \mathrm{C}$. Supernatants were aliquoted and kept at $-20^{\circ} \mathrm{C}$ until analyzed. Protein concentrations were measured with the NanoPhotometer Pearl (Implen). Lysates were pre-cleared with protein A/G agarose beads (Santa Cruz Biotechnology) for 1 hour at $4{ }^{\circ} \mathrm{C}$ with gentle rocking, centrifuged at $1000 \mathrm{~g}$ for $30 \mathrm{~s}$ and supernatants $(200 \mu \mathrm{l})$ incubated with either $1 \mu \mathrm{g}$ of antibody against P2X4 receptor (Alomone Labs, Jerusalem, Israel; APR-002), or $1 \mu \mathrm{g}$ of antibody against MFG-E8 (Santa Cruz Biotechnology; sc-217574) for 1 hour at $4{ }^{\circ} \mathrm{C}$ in rotating device. Then $20 \mu 1$ of $50 \% \mathrm{~A} / \mathrm{G}$ agarose beads slurry was added into the each vial and incubated at $4{ }^{\circ} \mathrm{C}$ overnight in the rotating device. Immunoprecipitates were collected by centrifugation at $1000 \mathrm{~g}$ for $30 \mathrm{~s}$ and supernatants washed 3 times with $1 \mathrm{ml}$ Pierce IP lysis buffer. After final wash pellets were resuspended in the Laemmli sample buffer, heated for $5 \mathrm{~min}$ at $95^{\circ} \mathrm{C}$ and loaded on Mini-PROTEAN TGX precast gels (Bio-Rad) for electrophoresis in Mini-PROTEAN Tetra cell apparatus (Bio-Rad). Gels were then blotted onto a PVDF membrane in a semi-dry Trans-Blot Turbo transfer system (Bio-Rad) and blocked for 1 hour at room temperature with 5\% BSA (Applichem) in PBS containing 0.18\% Tween-20 (PBS-Tw). The membranes were then probed with primary antibodies against MFG-E8 and $\mathrm{P} 2 \mathrm{X} 4$ receptor for 1 hour at room temperature. After incubation with primary antibodies membranes were washed three times in PBS-Tw. After washing, membranes were incubated further with horseradish peroxidase (HRP)-conjugated secondary antibody (Thermo Scientific) for 1 hour at room temperature. Washing procedure was repeated and immunoreactive bands were detected with Clarity ECL Western blotting substrate (Bio-Rad) using ChemiDoc MP system (Bio-Rad).

\section{Proximity ligation assay}

To detect protein - protein interactions between MFG-E8 and P2X4 receptor in the human microglial cells proximity ligation assay (PLA) was conducted using the Duolink ${ }^{\circledR}$ in situ detection kit (Sigma) according to the manufacturer's instructions. Microglial cells were treated with 1 AU of EVs for 30 min, 1 hour and 2 hours, then washed with PBS and fixed with 4\% PFA for 20 min at RT. After washing three times with PBS, cells were permeabilised with $0.1 \%$ Triton X-100 for 15 min, washed three times with PBS again, and blocked with Duolink blocking solution for 1 hour at $37^{\circ} \mathrm{C}$. Cells were then incubated with a pair of primary antibodies against MFG-E8 (Santa Cruz 
Biotechnology; sc-217574) and P2X4 receptor (Alomone Labs; APR-002) (dilution 1:500) for 1 hour at RT, washed two times for 5 min with wash buffer A and incubated with a mix of PLA antirabbit PLUS and PLA anti-mouse MINUS oligonucleotide - conjugated secondary antibodies for 1 hour at $37^{\circ} \mathrm{C}$. After ligation, amplification with polymerase and final wash in buffer B, coverslips were mounted onto the slides using Duolink ${ }^{\circledR}$ PLA Mounting Medium with DAPI and analyzed with with Leica TCS SP8 confocal microscope (Leica Microsystems, Mannheim, Germany). Images were taken using $63 \mathrm{x}$ oil immersion lens.

Quantification was performed with ImageJ program by counting numbers of PLA dots in each cell. The final result was evaluated by dividing the number of PLA dots by the number of cells (total number of cells in each microscope's field of view was determined by counting f DAPI-stained nuclei). Data were collected in two biological replicates and quantification of PLA dots between P2X4 and MFG-E8 proteins was performed in 8 independent fields of view for each experimental group.

\section{Inhibition of MFG-E8 receptor}

Human microglial cells were plated on rat tail collagen type I pre-coated 24-well plates (for ELISA assays) and on the glass coverslips (20 000 cells/well) for 72 hours in complete DMEM medium depleted of EVs (for lipid raft labeling). Microglial cells were pre-treated with $10 \mu \mathrm{M}$ of cilengitide (Sigma) for 2 hours and exposed to EVs (1 AU) for 30 min then lipid raft labeling was performed.

\section{Assessement of lipid raft formation}

Lipid rafts were labeled in live cells using Vybrant ${ }^{\circledR}$ Alexa Fluor ${ }^{\circledR} 594$ Lipid Raft Labeling Kit (Thermo Fisher Scientific, V-34405) according to the manufacturer's protocol with minimal modifications. Labeling is based on the specific interaction between fluorescent-labeled cholera toxin subunit B (CT-B) and ganglioside M1 (GM1), which is abundantly expressed in the lipid rafts. After all treatments, cells were washed once with complete growth medium, then incubated with $1 \mu \mathrm{g} / \mathrm{ml}$ fluorescent CT-B for 10 min at room temperature. After washing 3 times with PBS, fluorescent CTB-labeled lipid rafts were cross-linked with anti-CT-B antibody (200-fold dilution in complete growth medium) for $15 \mathrm{~min}$ at room temperature. Thereafter cells were washed 3 times with PBS and fixed in freshly prepared 4\% PFA for 20 min at room temperature. Cells were washed 3 times with PBS and coverslips mounted onto the slides using Duolink® PLA Mounting Medium with DAPI and analyzed with Leica TCS SP8 confocal microscope (Leica Microsystems, Mannheim, Germany). Images were taken using $63 \mathrm{x}$ oil immersion lens. 
Quantification was performed with Leica Application Suite X (LAS X) software (Leica Microsystems). The mean fluorescence intensity values taken from the each field of view were divided by the number of cells detected (determined by counting DAPI-stained nuclei). Data were collected from two biological replicates and quantification performed in 10 fields of view for each experimental group.

\section{Statistical analysis}

Statistical analysis was performed from data of at least three independent biological experiments. Graphs represent mean and standard deviation (SD) or standard error of the mean (SEM) values. Differences between 2 groups were compared by Student's t-test, while differences between three or more groups were compared by one-way ANOVA. Data, which did not passed the Shapiro-Wilk test of normality, were analyzed with non-parametric one-way ANOVA (Kruskal-Wallis one-way analysis of variance) using Dunn's multiple comparison post-hoc test. All results were considered as significant, when $\mathrm{p}<0.05$. Data analyzed using Graph Pad Prism ${ }^{\circledR}$ software version 8.0.1 (Graph Pad Software, Inc., USA).

\section{Acknowledgements}

This work was supported by funding from European Regional Development Fund (project No 01.2.2-LMT-K-718-01-0012) under grant agreement with the Research Council of Lithuania. KK was supported by the STSM Grant from European Commission COST Action (CA16122). TM is supported by Academy of Finland (328287) and Business Finland (4399/31/2019).

\section{Figure legends}

Figure 1. Characterization of extracellular vesicles (EVs) isolated from stem cells from the dental pulp of human exfoliated deciduous teeth (SHEDs). (A) Transmission electron microscopy of EVs isolated from SHEDs $(\times 120,000$ magnification $)$. (B) Determination of the 
concentration and particle size of EVs derived from SHEDs. Nanoparticle tracking analysis was performed with NanoSight LM10 instrument (Malvern Panalytical). Size distribution of the EVs was around $150 \mathrm{~nm}$. (C) Samples from cell lysates (L) and extracellular vesicles (EVs) were subjected to electrophoresis, blotted and the membrane was probed with antibodies against MFGE8. Bands were visualized by incubation with appropriate horseradish peroxidase-conjugated secondary antibodies and chemiluminescence substrate. Full blot is available in Supplementary Figure 1.

Figure 2. EVs increase intracellular $\mathrm{Ca}^{2+}$ in human microglial cells. (A) Experimental design. $(\mathrm{B}, \mathrm{C})$ Response of human microglial cells to acute 1 min treatment of EVs. Intracellular Ca2+ concentrations in single cells were visualized with the sensitive imaging system (Till Photonics, Germany). The peak of fluorescence in each individual cell during EV treatment was normalized to peak of fluorescence after response to ionomycin and presented as a percent of intracellular calcium influx. The graph represent mean \pm SEM, statistically significant difference was determined by Mann-Whitney test, ${ }^{* * * *} \mathrm{p}<0.0001$ ( $\mathrm{n}=85-127$ cells from 3 experiments).

Figure 3. EVs trigger ATP release from human microglial cells. Microglial cells were exposed to EVs (1 AU/well) diluted in BS for 20 and 60 minutes. Supernatants from each well were subjected to ATP assay. ATP was measured using ATPlite Luminescence Assay System with Perkin Elmer Wallac 1420 Victor2 instrument using Wallac 1420 software. Each bar represents mean \pm SEM, statistically significant difference was determined by two-way ANOVA, Sidak's multiple comparisons test, ${ }^{*} \mathrm{p}<0.05$ ( $\mathrm{n}=15-20$ wells from 3 experiments).

\section{Figure 4. EVs promote migration of microglia through the $\mathrm{P} 2 \mathrm{X} 4$ receptor - dependent}

mechanisms. (A) Migration assays were performed in the Boyden 10 well chemotaxis chamber (Neuro Probe) using $8 \mu \mathrm{m}$ pore polycarbonate membranes. Microglial cells were plated into the upper wells, pre-treated for 30 min with inhibitors against different subtypes of purinergic receptors, treated with $1 \mathrm{AU}$ of EVs and then left overnight. The number of cells that transmigrated to the opposite side of the membrane was determined using optical microscope in three randomly chosen fields from at least three wells for each experimental group. (B) EVs promote migration of human microglial cells. Data represent mean \pm SEM values, Welch's test, $* * * * \mathrm{p}<0.0001 ; \mathrm{n}=22$ (C) The effects of nonselective antagonist of ATP-gated P2 receptors suramin (200 $\mu \mathrm{M})$; (D) inhibitor of P2Y12 AR-C69931 (1 $\mu \mathrm{M})$; (E) selective inhibitor of P2X4R 5-BDBD (10 $\mu \mathrm{M})$; (F) ATP degrading enzyme apyrase $(20 \mathrm{U} / \mathrm{ml})$. For these experiments apyrase and $1 \mathrm{AU}$ of EVs were added simultaneously to the upper wells and cells incubated overnight. Data represent measurements from three independent experiments (only treatment with 5-BDBD from five). The 
graph represent mean \pm SD values, groups were compared with one-way ANOVA, Tukey's multiple comparisons test, ${ }^{*} \mathrm{p}<0.05, * * \mathrm{p}<0,01, * * * \mathrm{p}<0.001$.

Figure 5. EVs promote association between MFG-E8 and P2X4 receptor proteins in human microglia. (A) Confocal images of proximity ligation assay (PLA) dots (red) in human microglial cells. The cells were treated with $1 \mathrm{AU}$ of EVs for $30 \mathrm{~min}, 1 \mathrm{~h}$ and $2 \mathrm{~h}$ before PLA was conducted. Nuclei, DAPI (blue). Scale bar $=100 \mu \mathrm{m}$. (B) PLA dots per cell were counted with ImageJ program. Data shown represent results of 10 fields of view for each experimental group from three independent biological experiments Data represents mean $\pm \mathrm{SEM}, * \mathrm{p}<0.05 ; \mathrm{N}=3$; KruskalWallis, Dunn's multiple comparisons test.

Figure 6. Co-immunoprecipitation of MFG-E8 and P2X4. Representative western blots showing co-immunoprecipitation of MFG-E8 and P2X4 receptor proteins in human microglial cells. Full blots are available in Supplementary Figure 2.

Figure 7. Inhibition of MFG-E8 receptor with cilengitide suppressed EV - induced migration of microglia. For migration experiments (see above) microglial cells were pre-treated for $30 \mathrm{~min}$ with inhibitor MFG-E8 receptor cilengitide $(10 \mu \mathrm{M})$, then treated with 1 AU of EVs. Data represents mean $\pm \mathrm{SD}, * * \mathrm{p}<0.01,{ }^{* * *} \mathrm{p}<0.001 ; \mathrm{N}=3$; One way ANOVA, Tukey's multiple comparisons test.

Figure 8. Inhibition of MFG-E8 receptor with cilengitide suppressed EV - induced formation of lipid rafts. (A) Confocal images of lipid rafts (red) expressing human microglial cells. The cells were pre-treated with $10 \mu \mathrm{M}$ cilengitide for $2 \mathrm{~h}$ and then treated with EVs ( $1 \mathrm{AU}$ ) of EVs for 30 min before lipid raft labeling and fixation. Lipid rafts were labeled with Vybrant ${ }^{\circledR}$ Alexa Fluor ${ }^{\circledR} 594$ Lipid Raft Labeling Kit (Thermo Fisher Scientific) according to the manufacturer's protocol. Nuclei, DAPI (blue). Scale bar $=100 \mu \mathrm{m}$. (B) The mean fluorescence intensity of lipid rafts per cell were measured with Leica Application Suite X (LAS X) software. Data shown represent results of 15 fields of view for each experimental group from three independent biological experiments $(n=3)$ plotted as mean \pm SEM. Statistical significance was analyzed by Kruskal-Wallis test using Dunn's multiple comparison post-hoc test. ***, $\mathrm{xxx}, \mathrm{p}<0.001$;

Figure 9. Proposed mechanism for the EV action on microglial cells. EVs carrying MFG-E8 proteins associated with phosphatidylserine exposed on the outer membrane are recognized by the $\alpha \mathrm{V} \beta 3 / \alpha \mathrm{V} \beta 5$ integrin receptors of microglial cells and trigger lipid raft formation, interaction with P2X4 receptors and possibly other molecules enriched in the lipid rafts such as components of TLR4 
multireceptor complex. These events lead to the upregulation of intracellular $\mathrm{Ca} 2+$, release of ATP and increased motility of microglia.

Supplementary Figure 1. Western blot showing MFG-E8 expression levels in EVs derived from SHEDs.

Supplementary Figure 2. (A) Western Blot with Anti-P2X4 (IP P2X4), (B) Western Blot with AntiMFG-E8 (IP MFG-E8).

\section{References}

1. Salter, M.W. and B. Stevens, Microglia emerge as central players in brain disease. Nat Med, 2017. 23(9): p. 1018-1027.

2. Ransohoff, R.M., How neuroinflammation contributes to neurodegeneration. Science, 2016. 353(6301): p. 777-83.

3. Song, W.M. and M. Colonna, The identity and function of microglia in neurodegeneration. Nat Immunol, 2018. 19(10): p. 1048-1058.

4. Wiklander, O.P.B., et al., Advances in therapeutic applications of extracellular vesicles. Sci Transl Med, 2019. 11(492).

5. Alvarez-Erviti, L., et al., Delivery of siRNA to the mouse brain by systemic injection of targeted exosomes. Nat Biotechnol, 2011. 29(4): p. 341-5.

6. Guo, S., et al., Intranasal Delivery of Mesenchymal Stem Cell Derived Exosomes Loaded with Phosphatase and Tensin Homolog siRNA Repairs Complete Spinal Cord Injury. ACS Nano, 2019. 13(9): p. 10015-10028.

7. Saint-Pol, J., et al., Targeting and Crossing the Blood-Brain Barrier with Extracellular Vesicles. Cells, 2020. 9(4).

8. Long, Q., et al., Intranasal MSC-derived A1-exosomes ease inflammation, and prevent abnormal neurogenesis and memory dysfunction after status epilepticus. Proc Natl Acad Sci U S A, 2017. 114(17): p. E3536-E3545.

9. Zhuang, X., et al., Treatment of brain inflammatory diseases by delivering exosome encapsulated anti-inflammatory drugs from the nasal region to the brain. Mol Ther, 2011. 19(10): p. 1769-79.

10. Kim, D.K., et al., Chromatographically isolated CD63+CD81+ extracellular vesicles from mesenchymal stromal cells rescue cognitive impairments after TBI. Proc Natl Acad Sci U S A, 2016. 113(1): p. 170-5.

11. Narbute, K., et al., Intranasal Administration of Extracellular Vesicles Derived from Human Teeth Stem Cells Improves Motor Symptoms and Normalizes Tyrosine Hydroxylase Expression in the Substantia Nigra and Striatum of the 6-Hydroxydopamine-Treated Rats. Stem Cells Transl Med, 2019. 8(5): p. 490-499. 
12. Losurdo, M., et al., Intranasal delivery of mesenchymal stem cell-derived extracellular vesicles exerts immunomodulatory and neuroprotective effects in a $3 x T g$ model of Alzheimer's disease. Stem Cells Transl Med, 2020.

13. Perets, N., et al., Golden Exosomes Selectively Target Brain Pathologies in Neurodegenerative and Neurodevelopmental Disorders. Nano Lett, 2019. 19(6): p. 34223431.

14. Csoka, B., et al., Macrophage P2X4 receptors augment bacterial killing and protect against sepsis. JCI Insight, 2018. 3(11).

15. Zabala, A., et al., $P 2 X 4$ receptor controls microglia activation and favors remyelination in autoimmune encephalitis. EMBO Mol Med, 2018. 10(8).

16. Ohsawa, K., et al., Involvement of $\mathrm{P} 2 \mathrm{X} 4$ and $\mathrm{P} 2 \mathrm{Y} 12$ receptors in ATP-induced microglial chemotaxis. Glia, 2007. 55(6): p. 604-16.

17. Kobayakawa, K., et al., Macrophage centripetal migration drives spontaneous healing process after spinal cord injury. Sci Adv, 2019. 5(5): p. eaav5086.

18. Abiega, O., et al., Neuronal Hyperactivity Disturbs ATP Microgradients, Impairs Microglial Motility, and Reduces Phagocytic Receptor Expression Triggering Apoptosis/Microglial Phagocytosis Uncoupling. PLoS Biol, 2016. 14(5): p. e1002466.

19. Imura, Y., et al., Microglia release ATP by exocytosis. Glia, 2013. 61(8): p. 1320-30.

20. Ren, H., et al., Toll-like receptor-triggered calcium mobilization protects mice against bacterial infection through extracellular ATP release. Infect Immun, 2014. 82(12): p. 507685.

21. Dosch, M., et al., Mechanisms of ATP Release by Inflammatory Cells. Int J Mol Sci, 2018. 19(4).

22. Jonavice, U., et al., Extracellular vesicles can act as a potent immunomodulators of human microglial cells. J Tissue Eng Regen Med, 2019. 13(2): p. 309-318.

23. Ohsawa, K. and S. Kohsaka, Dynamic motility of microglia: purinergic modulation of microglial movement in the normal and pathological brain. Glia, 2011. 59(12): p. 1793-9.

24. Noda, M., et al., Calcium influx through reversed NCX controls migration of microglia. Adv Exp Med Biol, 2013. 961: p. 289-94.

25. Illes, P., et al., Regulation of Microglial Functions by Purinergic Mechanisms in the Healthy and Diseased CNS. Cells, 2020. 9(5).

26. Stokes, L., et al., P2X4 Receptor Function in the Nervous System and Current Breakthroughs in Pharmacology. Front Pharmacol, 2017. 8: p. 291.

27. Cheyuo, C., M. Aziz, and P. Wang, Neurogenesis in Neurodegenerative Diseases: Role of $M F G-E 8$. Front Neurosci, 2019. 13: p. 569.

28. Nakai, W., et al., A novel affinity-based method for the isolation of highly purified extracellular vesicles. Sci Rep, 2016. 6: p. 33935.

29. Kranich, J., et al., In vivo identification of apoptotic and extracellular vesicle-bound live cells using image-based deep learning. J Extracell Vesicles, 2020. 9(1): p. 1792683.

30. Wang, J., et al., MFGE8 mitigates brain injury in a rat model of SAH by maintaining vascular endothelial integrity via TIGbeta5/PI3K/CXCL12 signaling. Exp Brain Res, 2021.

31. Ren, Y., et al., Milk fat globule EGF factor 8 restores mitochondrial function via integrinmedicated activation of the FAK-STAT3 signaling pathway in acute pancreatitis. Clin Transl Med, 2021. 11(2): p. e295.

32. Miller, Y.I., et al., Lipid rafts in glial cells: role in neuroinflammation and pain processing. J Lipid Res, 2020. 61(5): p. 655-666.

33. Fuller, A.D. and L.J. Van Eldik, MFG-E8 regulates microglial phagocytosis of apoptotic neurons. J Neuroimmune Pharmacol, 2008. 3(4): p. 246-56.

34. Deroide, N., et al., MFGE8 inhibits inflammasome-induced IL-1beta production and limits postischemic cerebral injury. J Clin Invest, 2013. 123(3): p. 1176-81. 
35. Kopp, R., et al., P2X7 Interactions and Signaling - Making Head or Tail of It. Front Mol Neurosci, 2019. 12: p. 183.

36. Perez-Flores, G., et al., The P2X7/P2X4 interaction shapes the purinergic response in murine macrophages. Biochem Biophys Res Commun, 2015. 467(3): p. 484-90.

37. Boddaert, J., et al., Evidence of a role for lactadherin in Alzheimer's disease. Am J Pathol, 2007. 170(3): p. 921-9.

38. Allsopp, R.C., U. Lalo, and R.J. Evans, Lipid raft association and cholesterol sensitivity of P2X1-4 receptors for ATP: chimeras and point mutants identify intracellular aminoterminal residues involved in lipid regulation of P2X1 receptors. J Biol Chem, 2010. 285(43): p. 32770-32777.

39. Sezgin, E., et al., The mystery of membrane organization: composition, regulation and roles of lipid rafts. Nat Rev Mol Cell Biol, 2017. 18(6): p. 361-374.

40. Thery, C., et al., Isolation and characterization of exosomes from cell culture supernatants and biological fluids. Curr Protoc Cell Biol, 2006. Chapter 3: p. Unit 322. 